\title{
Sexual health in general practice: introductory skills
}

\author{
Philippa Matthews
}

General Practitioner,

Birmingham, UK

\section{Correspondence to Dr Philippa Matthews; p_m_matthews@hotmail.com}

Received 29 October 2010 Accepted 2 November 2010

\section{Sexual history taking}

General practice consultation is quite a lonely craft. In the early days of our training we share consultations - sitting in with our trainers, or having our trainers sit in with us. Then we have the company of the video camera and can share and discuss the highs, the excruciating moments - and the 'probably good enough' followed by decades of practice. A minority of the brave may turn again to the use of videoing, or may share occasional consultations with colleagues. Some of us talk through case histories and difficult consultations. But for almost all of us, almost all of the time, it is our four walls and the patient. Oh - and patient feedback questionnaires.

GPs hold a whole array of potential questions in their heads - questions to elicit important information in the assessment of, for example, respiratory, psychiatric or abdominal problems. However, for sexual history taking, for me, there was a blank space. I had been taught nothing. It took me several years to find out that being able to take a sexual history was quite useful. I had never seen another doctor use - and certainly not teach - this skill.

In teaching myself (and, at the time, finding myself needing to work out how to teach others) to take a sexual history, I found I first needed to establish a comprehensive list of stereotypical questions to draw on, such as I had for every other area of history taking. When I am assessing someone with depression, I will try to be as sensitive and empathic to the individual patient as I can, but it is striking how 'samey' the questions are that I use, the ways that I word them. Even the questions I use to assess suicide risk. I use the wording that I have learned works for me. However, it seemed to me that taking a sexual history required more adaptation and flexibility towards the individual patient than enquiring into other aspects of health, especially with its key component of the partner history. I also found that this area of history taking often required careful introduction in comparison with other areas, partly because the patient might not be expecting the doctor to raise the topic.

\section{Conscious competence}

As I gradually stumbled less and listened more, I realised I had what was now an essential skill. Some days I would take a sexual history four or more times. I had made the initial shift from unconscious incompetence to conscious incompetence. Then I had improvised my way through to conscious competence, at least some of the time, with unconscious competence finally freeing me to really listen to the answers and observe.

I still find many doctors and practice nurses have to make that classic educational journey to conscious competence as they learn about all different aspects of sexual health. The delight is that many find it intensely interesting - and even inspiring. Current training is undoubtedly better, but still has gaps. For young doctors for whom sexual history taking has only been taught by sexual health specialists, a major gap is the skill to introduce the topic of sexual health into the consultation when the patient may or may not be expecting it. A junior doctor in the practice (who had gained all sorts of qualifications relating to sexual health) had seen a 23-year-old man who had quite persistent mouth ulcers. Arming Dr U with the information that HIV should be on his list of diagnostic possibilities actually served him no use at all. How on earth was he to bring that subject up? He did not feel he could just leap into a history to assess HIV risk: what would be the first question? Another junior doctor saw a female health professional 
in her mid-40s with intermenstrual bleeding (IMB). Dr B probably had the skills to raise the subject of chlamydia with a younger woman with similar symptoms, but she found herself paralysed when faced with a woman she perceived as her senior - and a woman not apparently in a population risk group for chlamydia.

\section{Asking the patient}

It is of course always worth asking the patient themselves what they think the cause of their symptoms might be. However, I find that patients with symptoms where a sexually transmitted infection (STI) is on the list of differential diagnoses very rarely oblige by saying well actually they were wondering if it was HIV or chlamydia... Perhaps otherwise they would have gone to a genitourinary medicine (GUM) clinic (if they have one near enough), to form part of a service user profile that does differ from that in general practice. So our young man with mouth ulcers thought he might have a vitamin deficiency (because his mum had said that) and our woman thought her IMB was to do with her intrauterine device (IUD). The two young doctors are still faced with the task of assessing HIV or STI risk. They have become uncomfortably conscious of their incompetence.

There are some strategies they can use. "Mouth ulcers are a common problem, particularly in younger adults, and in 99\% of cases there is no underlying reason and they simply get better. However, I do always need to think about rare causes of common conditions, and mouth ulcers can occasionally be caused by HIV. Have you ever wondered if you could be at risk?” Dr U must note his patient's reaction and response, because what he says next will be guided by that. If his patient says "No, I don't think so" then Dr U will need to add "Can I ask you some questions to check?"

Dr B might say: "Bleeding between periods has a number of possible causes which we should look into carefully. I don't know if you are at risk or not, but one of these is chlamydia". Although a question has not been asked, Dr B will have useful verbal or nonverbal feedback at this point. Dr B might then add: "Could I ask you a few questions to see if you could be at risk?”

The given strategies are very similar, but will be differently nuanced by different doctors with different patients and different clinical presentations. But a core remains: the doctor is making it clear that the symptoms might be caused by an STI, but that it doesn't mean this is definitely the cause. They are also indicating that they are making no assumptions about the patient's level of risk but are interested to find out. Their cards are laid out on the table for the patient to see. The initial reaction of each patient will give the doctor important clues very quickly and these two consultations may be destined to be very different. However spending just a few seconds in this way will place both doctors in a good position to take a careful sexual history - and with a patient who is fully alert to the clinical significance of the process. They are heading for a conclusion where the patient will be well informed to give valid consent to testing, if appropriate. Dr U's pulse rate - and his patient's - is likely to be a little higher than those in Dr B's consulting room. But this is not guaranteed, because much depends on the individual patients and their personal, very personal, stories.

\section{Consultation comfort zones}

Some doctors faced with similar situations will entirely fail to raise the topic of sexual health. This may be because, unlike our two young doctors, they do not realise which symptoms or conditions might be caused by an STI. There is evidence that this is the case for patients attending general practice for a range of conditions, including, for example, HIV and epididymo-orchitis. Or it may be that they have wondered about an STI but simply do not feel they can raise the subject; they may comfort themselves with the thought that shingles isn't usually associated with HIV. And so the consultation remains firmly in the comfort zone of the doctor and, quite possibly, the patient.

Now imagine that these two patients, one with their mouth ulcers and one with their IMB, are asked to complete patient feedback questionnaires at the end of the consultation. Who is to say if the responses would be 'better' or 'worse' depending on whether they saw a doctor who avoided the topic of sexual health - or a doctor prepared to stretch and test their own skills? It is, of course, possible that the patient left 'undisturbed' would give better feedback - but patients often assume that the doctor will bring up the questions that need to be asked. However, I would still reserve the right to argue that the doctor who introduced the subject of sexual health into the consultation was, at the very least, heading for being the better doctor and not missing out important parts of taking a history.

If you would like to learn more about sexual history-taking in general practice, see e-GP on http:// www.e-GP.org.uk - sessions 11_001 through 11_003.

\section{Future articles}

Future articles in this mini-series will focus on specific topics relevant to sexual health in general practice.

\section{Competing interests None.}

Provenance and peer review Commissioned; internally peer reviewed. 


\section{Correction}

There was an error in an author's affiliation and contact e-mail address in an article published in the January 2011 issue of the journal (Sexual health in general practice: introductory skills. J Fam Plann Reprod Health Care 2011;37:8-9. doi:10.1136/ jfprhc.2010.0035). The author's affiliation should have been given as London (not Birmingham) and her current e-mail address is philippa.matthews@nhs.net.

J Fam Plann Reprod Health Care 2011;37:115. doi:10.1136/jfprhc.2010.0035.corr1 